\title{
Arthroscopic Osteochondral Grafting for Radiocarpal Joint Defects
}

\author{
Pak-cheong Ho, FHKCOS, FHKAM (Orthopaedic) ${ }^{1}$ Wing-lim Tse, FHKCOS, FHKAM (Orthopaedic) ${ }^{1}$ \\ Clar Wing-Yee Wong, FHKCOS, FHKAM (Orthopaedic) ${ }^{1}$ \\ Esther Ching-San Chow, FHKCOS, FHKAM (Orthopaedic) ${ }^{1}$
}

1 Division of Hand and Microsurgery, Department of Orthopaedic and
Traumatology, Prince of Wales Hospital, Chinese University of Hong
Kong, Hong Kong SAR

J Wrist Surg 2013;2:212-219.
Address for correspondence Pak-cheong Ho, FHKCOS, FHKAM (Orthopaedic), Division of Hand and Microsurgery, Department of Orthopaedic and Traumatology, Prince of Wales Hospital, Chinese University of Hong Kong, Hong Kong SAR (e-mail: pcho@ort.cuhk.edu.hk).
Focal articular cartilage damage is a common cause of persistent joint pain after articular or periarticular injury. It is frequently reported in the lower extremity, as in the knee joint and ankle joint, with or without an associated soft tissue lesion. Focal disruption of the articular cartilage can lead to secondary osteoarthritis. Restoration of the normal hyaline cartilage is often the goal of treatment. Over the last two decades, various techniques have been developed to repair cartilage defects in the lower extremity, including open or arthroscopic abrasion chondroplasty, microfracture, osteochondral autograft transfer, osteochondral allograft, and autologous chondrocyte implantation, with variable success. Focal chondral lesion is also a cause of chronic pain in the wrist, especially after trauma. ${ }^{1}$ The incidence remains
Copyright @ 2013 by Thieme Medical Publishers, Inc., 333 Seventh Avenue, New York, NY 10001, USA. Tel: +1(212) 584-4662.
DOI http://dx.doi.org/ $10.1055 / \mathrm{s}-0033-1351788$. ISSN 2163-3916. 
uncertain because of the difficulty in detecting these lesions with imaging studies in this small and complex joint. ${ }^{2}$ With a wider use of wrist arthroscopy, the diagnosis is more apparent. There is still, however, a paucity of treatment options for focal chondral lesion. Whipple recommended arthroscopic drilling for small chondral defects of $5 \mathrm{~mm}$ or less. ${ }^{3}$ For larger lesions, the best treatment method remains unknown. We performed an arthroscopic-assisted implantation of an osteochondral autograft from the knee joint in four patients for the treatment of of focal chondral defect in the distal articular surface of the radius with satisfactory clinical results.

\section{Patient Series}

Between December 2006 and December 2010, we operated on four patients with a posttraumatic localized osteochondral lesion over the dorsal aspect of the lunate fossa of the distal radius. There were three male patients and one female patient with an average age of 31 years (range $24-41$ years). The affected sides were the dominant right wrists. They all presented with chronic central dorsal wrist pain and loss of motion after an injury. The average duration of symptoms prior to surgery was 28.3 months (range 11-71 months). All patients had preoperative imaging, including a computed tomography (CT) scan, magnetic resonance imaging (MRI), or both. The definitive diagnosis was confirmed by arthroscopy. In the first three cases, the lesions were located at the dorsal lunate fossa measuring $6 \times 8 \mathrm{~mm}, 4 \times 8 \mathrm{~mm}$, and $4 \times 6 \mathrm{~mm}$, respectively. In the last patient, the lesion extended from the dorsal to the central lunate fossa and measured $8 \times 10 \mathrm{~mm}$. We performed an arthroscopic transplantation of an autogenous osteochondral graft taken from the knee. In three patients, one single osteochondral plug $6 \mathrm{~mm}$ in diameter was implanted, while in the last case, two osteochondral grafts were implanted. Two patients underwent additional procedures for other associated pathology in the wrist, including an arthroscopic-assisted reconstruction of the scapholunate ligament with a tendon graft in one patient and an ulnar shortening osteotomy plus an arthroscopic repair of a torn ulnocarpal ligament in another patient.

All patients were assessed by an occupational therapist before the index procedures, during and at the final followup. The range of motion (ROM) of the wrist, grip strength, wrist functional performance score, pain score, and return to work status were charted. The wrist functional performance score developed by our hospital was modeled on the findings of D. L. Nelson. ${ }^{4}$ It consists of ten common standardized tasks of activities of daily living (ADL) to be performed by the patient under the scrutiny of an occupational therapist (-Table 1) ${ }^{5,6}$ The performance on each task was rated by the therapist according to a 4-point scale, giving a maximum total of 40 for a normal performance of the complete test (-Table 2). A pain score on a 3-point scale was rated by the patient according to the pain level perceived during the performance of each ADL task. The total score ranged from zero to a maximum pain level of 20 points (-Table 3 ). Postoperative CT scan or MRI was performed to assess incorporation of the osteochondral graft. X-ray was used to moni-
Table 1 Wrist functional performance score

\begin{tabular}{|l|l|}
\hline Tasks & $\begin{array}{l}\text { Wrist motion and } \\
\text { grip required }\end{array}$ \\
\hline Wash back & F, S, Rd, Ud, Pinch \\
\hline $\begin{array}{l}\text { Pour water from } \\
\text { a full water pot }\end{array}$ & F, Ud \\
\hline Open gate & E, S \\
\hline Open jar lid & Ud, Rd, Grasp \\
\hline Hold a wok & E, S, Ud, Grasp \\
\hline Wring towel & F, E, P, S, UD, RD, Grasp \\
\hline Pull out drawer of 7 lbs & S, Grasp \\
\hline Lift weight of 5 lbs & E, Ud, Grasp \\
\hline Write name & E,Ud, Pinch \\
\hline Turn door knob & P,S \\
\hline
\end{tabular}

Abbreviations: E, extension; F, flexion; P, pronation, Rd, radial deviation; S, supination; Ud, ulnar deviation.

Table 2 Performance Score

\begin{tabular}{|l|}
\hline Performance Score (maximal total score $=\mathbf{4 0}$ ) \\
\hline 4 Can do without difficulty \\
\hline 3 Can do with minimum difficulty or frustration and with \\
satisfactory outcome \\
\hline 2 Can do only with some modification of activity; \\
it is awkward and frustrating \\
\hline 1 Frustrating to do and quality is poor; would stop doing \\
it most of the time \\
\hline 0 Cannot do \\
\hline
\end{tabular}

tor degenerative change. In three patients, a second-look arthroscopy was performed to evaluate the status of the chondral graft directly. A biopsy was performed in one case.

\section{Surgical Technique}

In all patients, diagnostic arthroscopy under local anesthesia was performed prior to the index procedure to evaluate the cause of the chronic wrist pain and to confirm the location of the osteochondral lesion.

The osteochondral grafting was performed under general anesthesia on a separate day after an informed consent was obtained from the patient. The patient was placed in the supine position with the operated arm supported on a hand table. A vertical joint traction force of 4-6 $\mathrm{kg}$ was applied

Table 3 Pain Score

\begin{tabular}{|l|}
\hline Pain Score $($ maximal total score $=\mathbf{2 0}$ ) \\
\hline 2 Intolerable pain in doing \\
\hline 1 Tolerable pain in doing \\
\hline 0 Pain free in doing \\
\hline
\end{tabular}




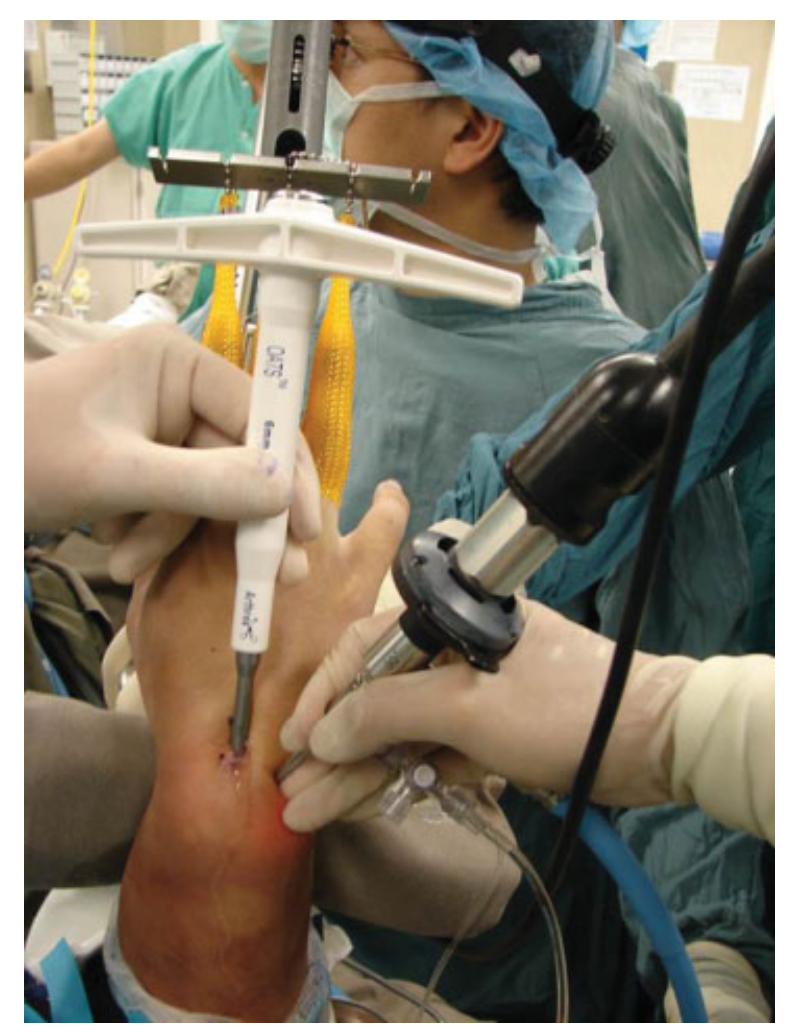

Fig. 1 A 6-mm trephine of OATS was inserted into the osteochondral defect through the 3-4 portal. The wrist was flexed to allowed easy passage. The arthroscope was placed at the $4-5$ portal to monitor the process.

through a wrist traction tower. We employed continuous saline irrigation of the joint with a $3 \mathrm{~L}$ bag of normal saline instilled under gravity.

A diagnostic inspection of the radiocarpal joint was performed with a $2.7-\mathrm{mm}$ arthroscope introduced through the 3-4 portal. Synovitis over the dorsal aspect of the lunate fossa was débrided with a $2.0-\mathrm{mm}$ shaver and radiofrequency probe inserted from the $4-5$ portal to uncover the underlying osteochondral lesion. The true extent of the lesion was measured using a probe. The proximal aspect of the lunate articular surface was assessed. Excessive cartilage damage is a contraindication to the use of the osteochondral graft.

The arthroscope was then placed in the 4-5 portal. We used the Osteoarticular Transfer System (OATS; Arthrex, Naples, FL) for both harvest and graft implantation. The 3-4 portal was gently dilated with a small hemostat, and a 6-mm recipient harvester with the obturator from the OATS set was carefully inserted into the joint. The wrist was passively flexed to $\sim 50^{\circ}$ while still in traction ( - Fig. 1). This maneuver moves the dorsal lip of the lunate palmarward and opens up the space between the lunate and the lunate fossa to accommodate the harvester. With the harvester positioned vertically and perpendicular to the osteochondral lesion at least $2 \mathrm{~mm}$ from the dorsal articular margin, the trephine was driven into the osteochondral defect for $10-12 \mathrm{~mm}$ while the depth gauge was observed arthroscopically (-Fig. 2). A cylindrical plug of bone containing the osteochondral defect was then removed.

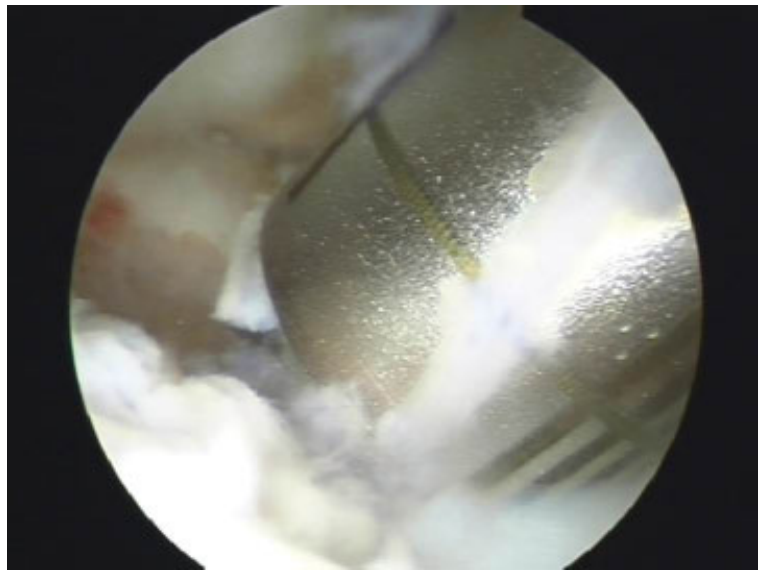

Fig. 2 Arthroscopic view of the trephine over the osteochondral defect at the dorsal lunate fossa.

The donor site was the knee of the nondominant leg. The graft was harvested according to the technique described by Hangody et al. ${ }^{7}$ Through a small incision, a $6-\mathrm{mm}$ donor harvester was inserted in the sulcus terminalis of the lateral femoral condyle to a depth slightly longer than that in the wrist ( - Fig. $\mathbf{3}$ ). The defect in the knee could partially be replaced by the cancellous bone obtained from the recipient site.

A short transparent plastic delivery tube was attached to the tip of the donor harvester containing the osteochondral plug. It was then gently inserted into the defect through the 3-4 portal with the aid of a rotating dial ( - Fig. 4). Graft

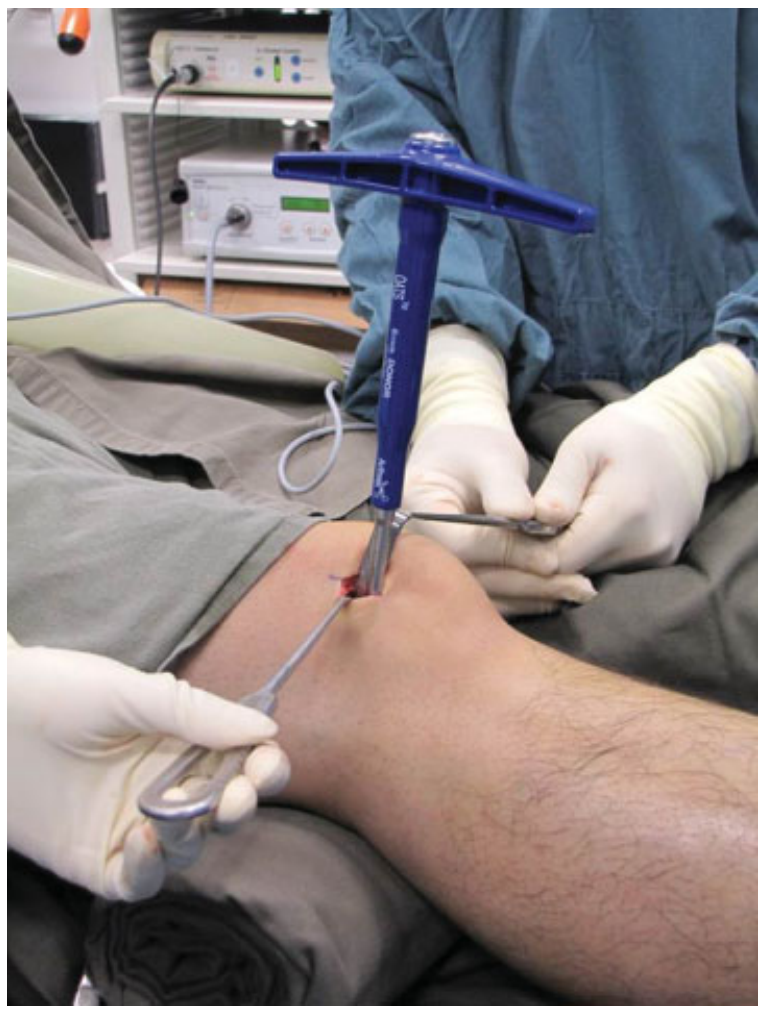

Fig. 3 6-mm OATS harvester was inserted to the lateral femoral condyle to harvest the osteochondral graft. 


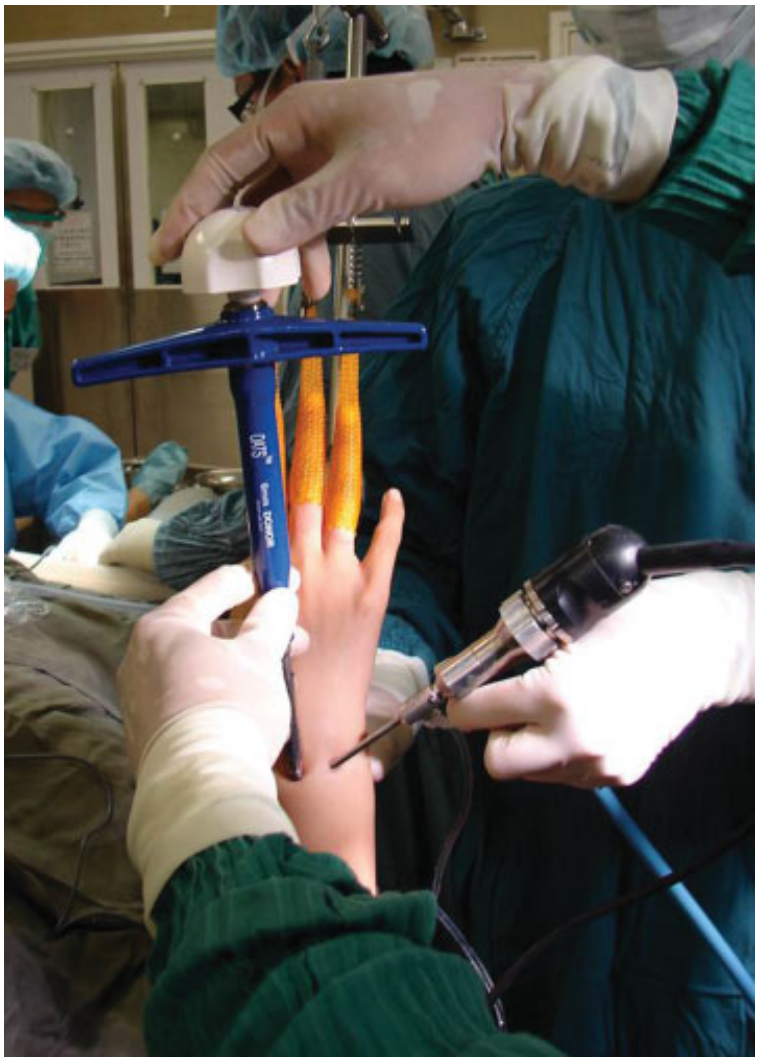

Fig. 4 The 6-mm OATS harvester with the osteochondral graft on was inserted into the wrist joint through the 3-4 portal. The graft was delivered into the defect with the dialed pusher.

insertion was monitored through the plastic sheath (-Fig. 5). The harvester was removed when the graft was flush with the articular surface. A plastic tamp was used to press-fit the plug. Any articular step-off was trimmed with an arthroscopic knife. The graft was generally very stable, and no internal fixation was required.

The wounds were apposed with adhesive surgical tape strips, followed by a bulky compressive dressing. A wrist

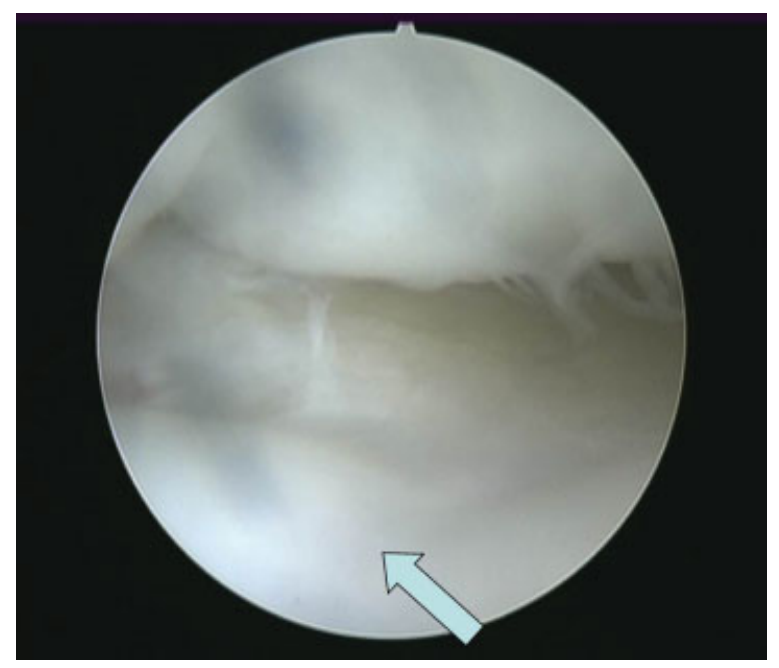

Fig. 6 A third-look arthroscopy at 29 months postoperative in a patient showed a complete incorporation of the hyaline cartilage graft (arrow) with minimal junctional defect.

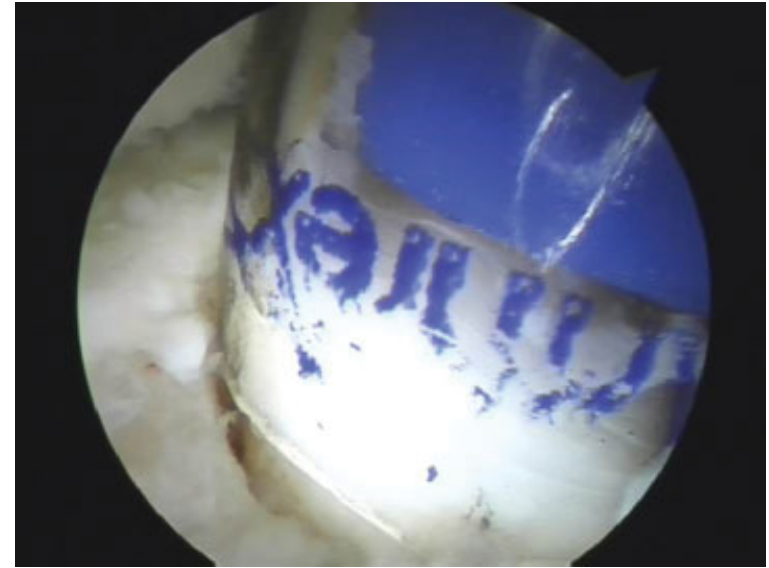

Fig. 5 Arthroscopic view of the osteochondral graft delivered into the defect through a transparent plastic tube.

splint was used for 2-4 weeks, and the patient was instructed to perform gentle active wrist motion from day 3. Passive motion was instituted at 4-6 weeks. Active use of the hand in self-care and daily activities was allowed, but load bearing of the wrist was discouraged for the first 6 weeks.

\section{Result}

In all cases, the osteochondral grafts were shown by CT scan or MRI to have completely incorporated in the host bone by 3-4 months postoperative. A second-look arthroscopy at 6-9 months postoperative in three patients confirmed the preservation of normal articular cartilage in the grafted area. There was a 1-2-mm rim defect between the graft and the host cartilage. A biopsy of the grafted area in one patient confirmed viable hyaline cartilage. In one patient, a third-look arthroscopy at 29 months postoperative showed complete coverage of the junction defect by cartilage (-Fig. 6).

At the final follow-up of average 48.5 months (range 24-68 months), all patients showed an improvement in the wrist performance score (preoperative $27.5 \pm 6.4$, postoperative $39.0 \pm 1.7$ on a 40 -point scale) and pain score (preoperative $9.5 \pm 2.2$, postoperative $0.5 \pm 0.9$ on a 20 -point scale). Grip strength improved from $62.6 \pm 9.0 \%$ to $98.2 \pm 7.6 \%$ of the contralateral side. Wrist motion improved from a flexion/ extension arc of $115.5 \pm 28.8^{\circ}$ to $131.3 \pm 23.6^{\circ}$. Two patients returned to their previous occupation, while the other two other patients could not do so for other medical reasons. They were all satisfied with the procedure. The surgical scars were inconspicuous. Followup X-ray showed good graft incorporation, with no joint space narrowing or other sign of degeneration. There was no complication of infection or tendon or nerve injury in this series.

\section{Case Illustration}

A 28-year-old man sustained a fracture of his right distal radius during a basketball game in 1993, when he was 15 years old. He was treated with a plaster cast, and the fracture healed uneventfully. He presented to us in 2000 with 


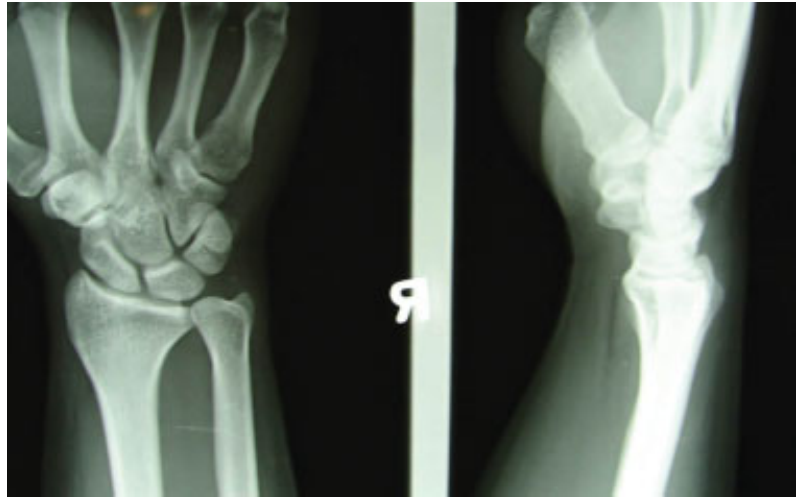

Fig. 7 X-ray of the right wrist of the patient in the case study showed no significant abnormality with normal joint space.

increasing, poorly localized wrist pain, easy fatigue, and coarse crepitus over the dorsal central part of his right wrist that interfered with his work as a mechanic. X-rays did not reveal any articular abnormality (-Fig. 7). A CT scan showed a possible osteochondral lesion over the dorsal lunate fossa (-Fig. 8).

Wrist arthroscopy in September 2000 revealed a localized $6 \times 8 \mathrm{~mm}$ osteochondral defect over the dorsal aspect of the lunate fossa, accompanied by localized synovitis and a type $1 \mathrm{a}$ triangular fibrocartilage complex (TFCC) tear (-Fig. 9). The synovitis and TFCC tear were débrided. The osteochondral defect was drilled. However, the joint pain and crepitus persisted, and he failed a second arthroscopic débridement in February 2003. It was also noted that a corresponding kissing chondral lesion measuring $2 \times 6 \mathrm{~mm}$, of Outerbridge grade 3-4, had developed over the proximal aspect of the lunate. Grip strength was reduced to $20 \mathrm{kgf}$ or $50 \%$ of the unaffected side. The combined extension/flexion range of the wrist was $130^{\circ}$. His wrist performance score was 34 out of 40 and pain score was 6 out of 20. Finally, in December 2006, an arthroscopic-assisted 6 - $\mathrm{mm}$ diameter $\times 15-\mathrm{mm}$ long osteo-

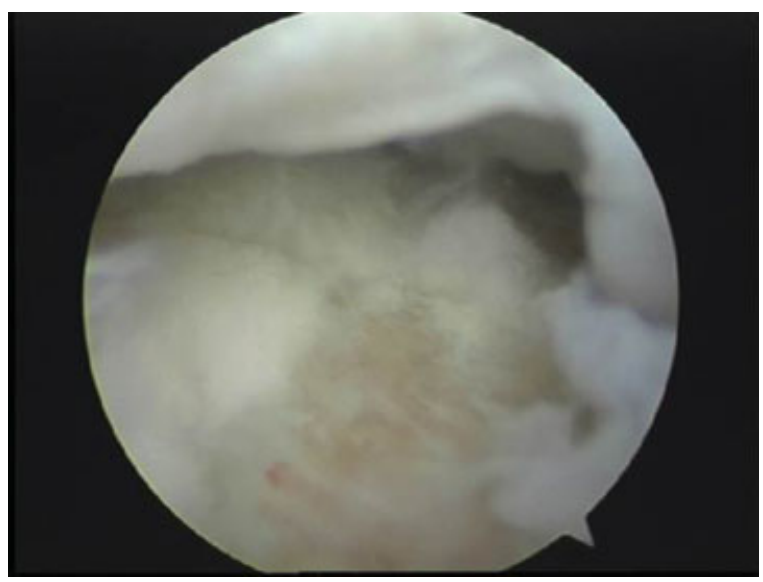

Fig. 9 Arthroscopy confirmed a full thickness focal osteochondral lesion of size $6 \times 8 \mathrm{~mm}$ over the dorsal lunate fossa.

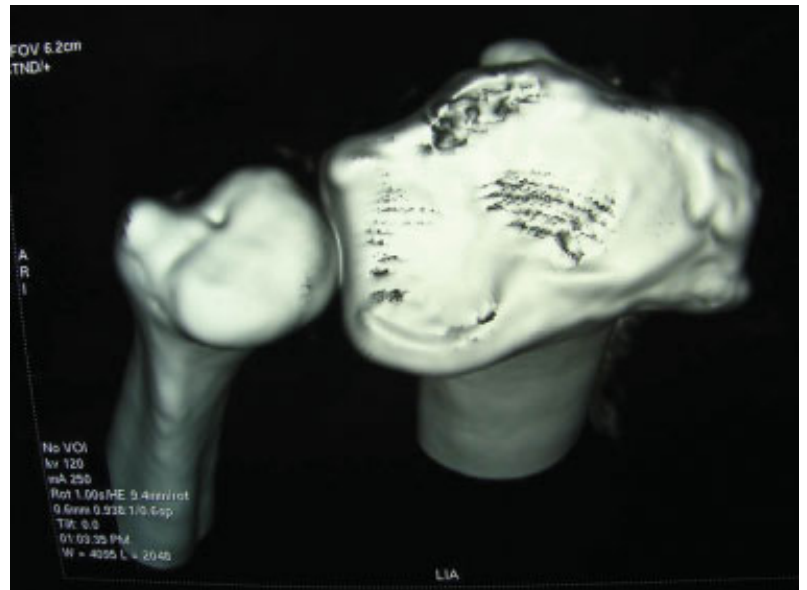

Fig. 8 CT scan showed possible osteochondral lesion over the dorsal lunate fossa.

chondral autograft, harvested from the left knee lateral femoral condyle, was implanted to repair the osteochondral defect (-Fig. 10).

He had no pain 2 months after the surgery. A MRI scan of his right wrist at 3 months postoperative showed good incorporation of the graft (-Fig. 11). A second-look arthroscopy under portal-site local anesthesia at 9 months revealed a stable osteochondral graft with preserved cartilage (-Fig. 12). There was a marginal increase in the junctional cleft between the graft and host cartilage. The kissing lesion over the proximal lunate remained unchanged. At the 68months postoperative follow-up, he had no pain except for a mild ache after prolonged work. He maintained his job as a mechanic. X-ray images showed a normal radiolunate joint space ( - Fig. 13). A grind test of his wrist did not reveal any joint crepitus. The grip strength increased to $50 \mathrm{kgf}$ or $104.2 \%$ of the unaffected side. The combined extension/flexion range of the wrist was $140^{\circ}(\boldsymbol{- F i g}$. 14). The surgical scar was inconspicuous (-Fig. 15). His wrist performance score was $40 / 40$, and pain score was $0 / 20$. He had no knee pain at the donor site.

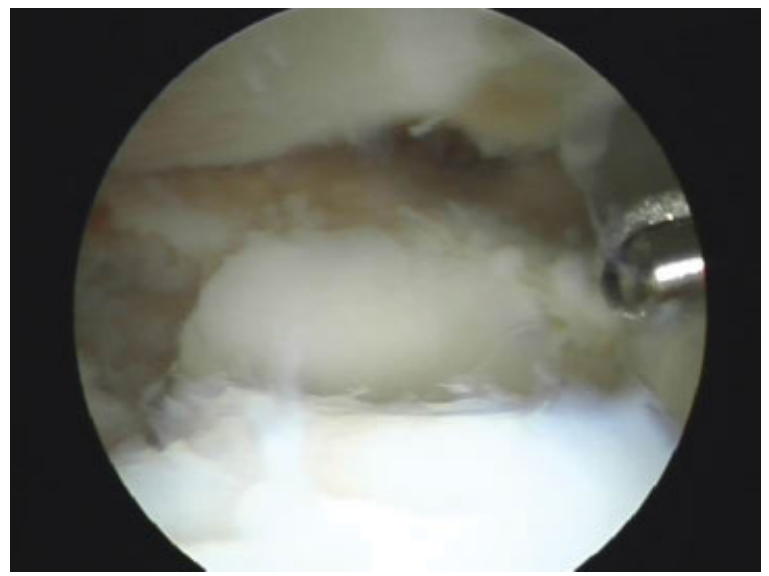

Fig. 10 A 6-mm $\times 15-m m$ osteochondral graft transplanted from the left knee was filling up the defect. 


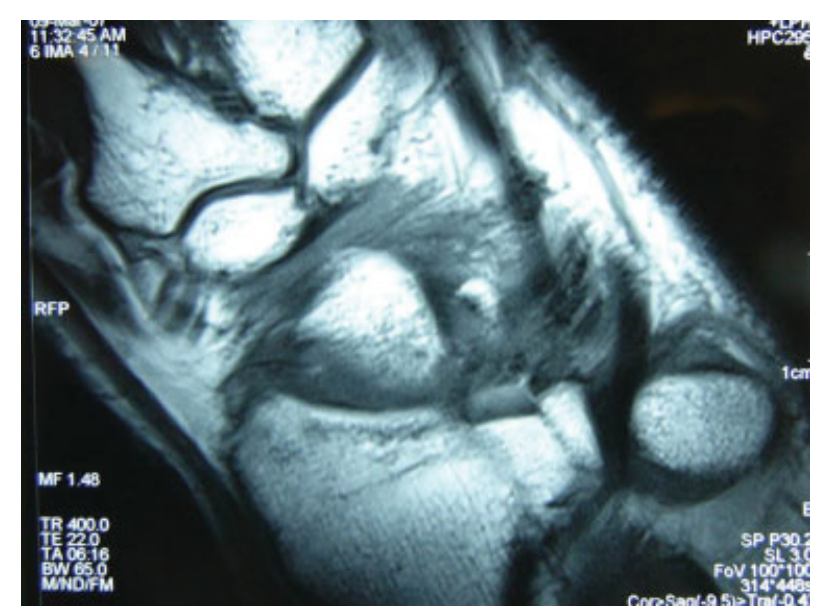

Fig. $11 \mathrm{MRI}$ of the right wrist at 3 months postoperative showed a good incorporation of the graft.

\section{Discussion}

Cartilage lesions seldom heal spontaneously. Articular cartilage is an avascular and aneural soft tissue. The cellular components contribute to less than $10 \%$ of the tissue volume. Given the lack of an undifferentiated cell pool within the cartilage substance and the low mitotic activity of chondrocytes in the physiological status, the healing of even a small cartilage lesion can be extremely difficult. ${ }^{8}$ Cartilage defects gradually lead to degeneration of the articular cartilage and osteoarthritis.

Various surgical strategies have been developed in the last two decades trying to overcome this biological hurdle. These techniques have included Pridie drilling, ${ }^{9}$ abrasion arthro-

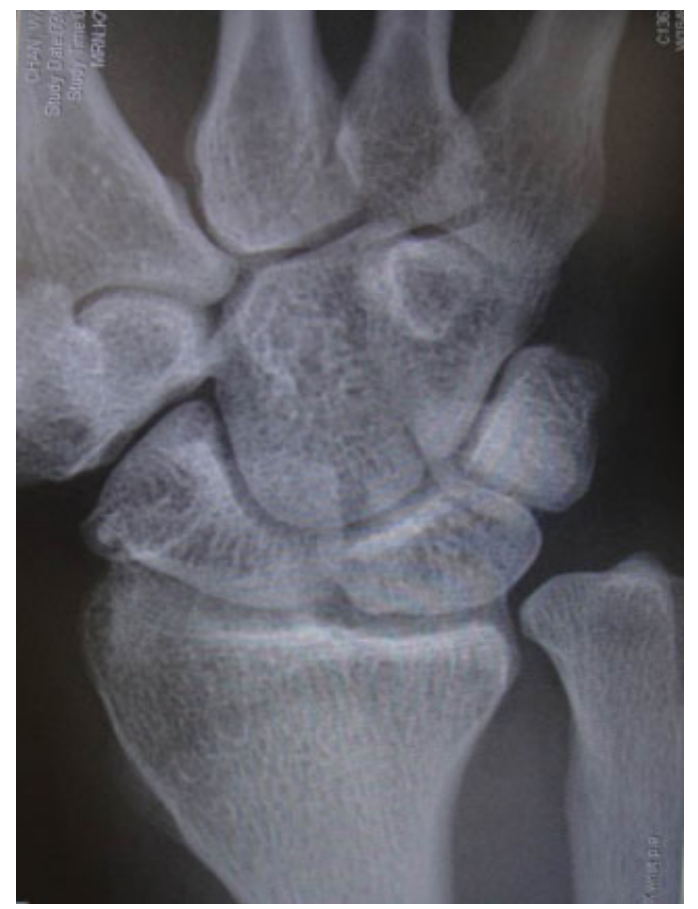

Fig. 13 At 68 months postoperative, X-ray showed normal joint space at radiolunate interval.

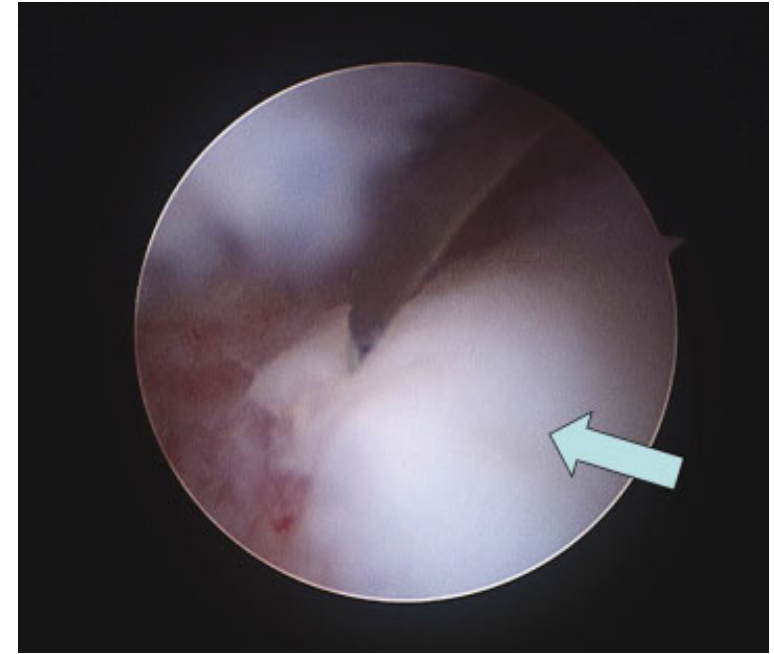

Fig. 12 A second look arthroscopy at 12 months post-operation showed a completely normal hyaline cartilage (arrow) at the grafted site surrounded by small zone of junctional defect.

plasty, and Steadman microfracture, ${ }^{10}$ with an aim to produce blood clots at the subchondral bone, which in turn would promote fibrocartilagenous regeneration. The clinical outcomes of these methods varied and were unpredictable. Other strategies included resurfacing with periosteal or perichondral grafts, ${ }^{11}$ osteochondral autograft transplantation, ${ }^{7}$ osteochondral allograft transfer, ${ }^{12}$ and autologous chondrocyte implantation. The American Academy of Orthopaedic Surgeons recently published recommendations for treating chondral defects in the tibiofemoral joint. ${ }^{13}$ They suggested use of osteochondral autograft for very small defects of $<2 \mathrm{~cm}^{2}$, microfracture for defect between 2 and $4 \mathrm{~cm}^{2}$, and autologous chondrocyte implantation (ACI) or osteochondral allograft for defect $>4 \mathrm{~cm}^{2}$.

The optimal treatment for chondral lesion in the wrist is controversial. This lesion has been recognized, however, as a common cause of wrist pain. It may result from osteochondral fractures, chronic carpal instability, or chondromatosis, or it may occur idiopathically. ${ }^{14}$ Cheng, in reviewing the surgical treatment on 22 patients with chronic pain post distal radius fracture treatment, noted chondral lesions in $63.6 \%$ of them. Fifty percent of them involved the radiocarpal joint and occurred at the distal radius. ${ }^{5}$ Culp et al described a modified Outerbridge classification for chondral lesion in the wrist and recommended débridement and synovectomy for grade I-III lesions. ${ }^{15}$ Grade IV lesions are treated with abrasion chondroplasty and subchondral drilling. Whipple reported a significant relief of symptoms in a small series following abrasion chondroplasty if the defect was smaller than $5 \mathrm{~mm}$ in size. ${ }^{3}$ However, there are very few studies on the treatment of larger chondral lesions in the wrist.

Small osteochodral autografts were used first for articular defects in the hand and more recently in the wrist. ${ }^{16-19}$ Boulas et al first reported the use of osteochondral metatarsophalangeal autografts for traumatic articular metacarpophalangeal defects in five patients in $1993 .^{16}$ In the wrist 


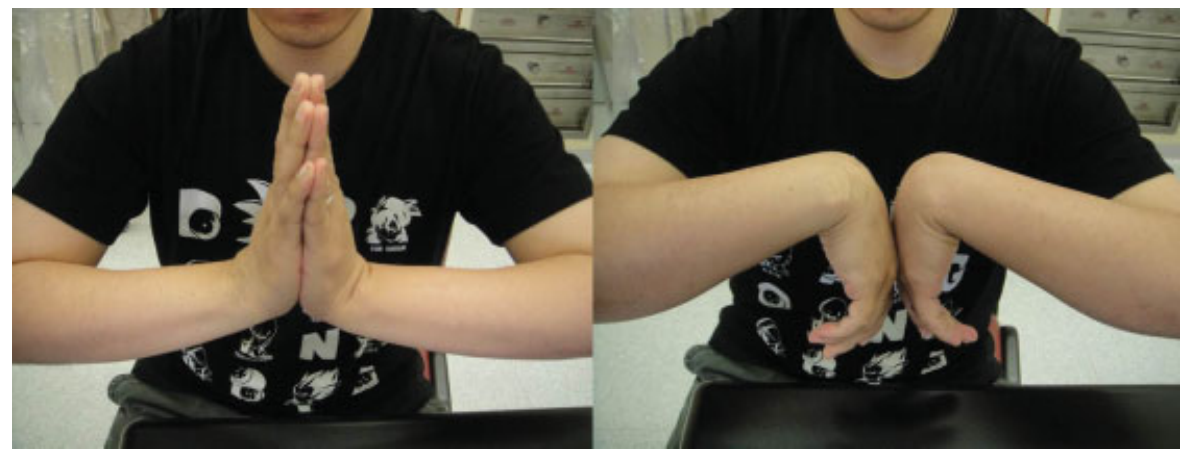

Fig. 14 The patient has good combined extension/flexion range of the wrist of $140^{\circ}$ with no pain.

region, Mehin et al reported two cases of autogenous osteochondral graft transfer from the proximal tibiofibular joint to resurface the distal radius in $2000 .{ }^{20}$ Merrell et al described a technique of using the scaphoid facet of the same wrist as a nonvascularised osteochondral graft to reconstruct the sigmoid fossa in one patient in 2002. ${ }^{21}$ In 2005 del Piñal et al published the case report on the use of a free vascularized osteochondral autograft from the second toe to reconstruct a defect involving $70 \%$ of the scaphoid facet of the distal radius in a 33-year-old male patient. ${ }^{22}$ In 2011 Lee et al reported a case of osteochondritis dissecans of the scaphoid treated with an osteochondral autograft. ${ }^{23}$. They used OATS to harvest a 6-mm graft from the lateral femoral condyle, which was press-fitted into the defect. There is no previous published report on an arthroscopic-assisted osteochondral graft as yet.

In our series, the indication for an arthroscopic-assisted autogenous osteochondral graft included the highly localized nature of the osteochondral defect, all involving the lunate fossa. Extreme caution had to be exercised to avoid iatrogenic injury to the unaffected articular surface. This procedure may not be technically possible with defects involving central or more volar lesions, since it is very difficult or impossible to insert the instruments perpendicular to the articular surface. The technique is also contraindicated for diffuse or progressive cartilage disease and in the presence of active sepsis. In

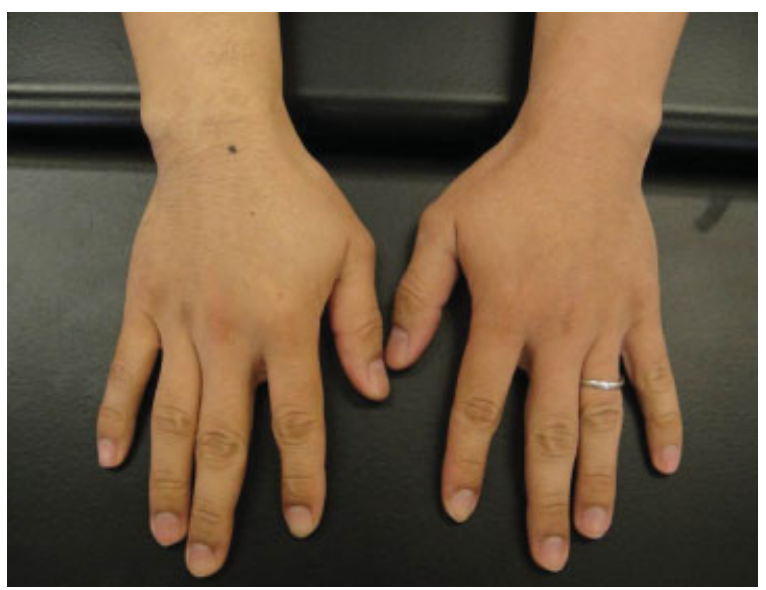

Fig. 15 Inconspicuous scar over the right wrist. our series, there was no major complaint about pain at the donor site at the knee. This is probably due to the small size of the graft and the limited donor site defect created by utilizing only one or two osteochondral plugs, in contrast to the experience in the knee or ankle, where multiple plugs are generally required.

The rehabilitation program will be affected by any concomitant procedures. For a stable isolated press-fitted osteochondral graft, we consider strict immobilization of the wrist unnecessary and allow early active and passive wrist motion. Load bearing, however, should be avoided until there are clinical and radiological signs of graft incorporation, usually by $2-4$ months postoperative.

In our experience an arthroscopic-assisted osteochondral autograft transfer is an effective option for the treatment of radiocarpal articular defects of over $5 \mathrm{~mm}$ in diameter. Due to limitation by the current instrumentation, the size of the osteochondral plug is restricted to $6 \mathrm{~mm}$. In the future, more patients may be benefited if the instrument can be downsized so that multiple grafting is made possible. A larger patient sample and long-term outcomes are necessary to establish the effectiveness of this procedure.

\section{Work Location}

Department of Orthopaedic and Traumatology, Prince of Wales Hospital, Chinese University of Hong Kong, Hong Kong SAR

\section{Conflict of Interest None}

\section{References}

1 Koman LA, Poehling GG, Toby EB, Kammire G. Chronic wrist pain: indications for wrist arthroscopy. Arthroscopy 1990;6(2):116-119

2 Haims AH, Moore AE, Schweitzer ME, et al. MRI in the diagnosis of cartilage injury in the wrist. Am J Roentgenol 2004;182(5): 1267-1270

3 Whipple TL. Chronic wrist pain. Instr Course Lect 1995;44: 129-137

4 Nelson DL. Functional wrist motion. Hand Clin 1997;13(1):83-92

5 Cheng HS, Hung LK, Ho PC, Wong J. An analysis of causes and treatment outcome of chronic wrist pain after distal radial fractures. Hand Surg 2008;13(1):1-10 
6 Estrella EP, Hung LK, Ho PC, Tse WL. Arthroscopic repair of triangular fibrocartilage complex tears. Arthroscopy 2007;23 (7):729-737, e1

7 Hangody L, Ráthonyi GK, Duska Z, Vásárhelyi G, Füles P, Módis L. Autologous osteochondral mosaicplasty. Surgical technique. J Bone Joint Surg Am 2004;86-A(Suppl 1):65-72

8 Buckwalter JA. Articular cartilage: injuries and potential for healing. J Orthop Sports Phys Ther 1998;28(4):192-202

9 Pridie EA. A method of resurfacing osteoarthritic knee joints. J Bone Joint Surg Br 1959;41:618

10 Steadman R. Chondral defects in athletes. The 5th Annual Panther Sports Medicine Symposium, Pittsburgh, PA, Apr 2-4, 1992

11 Engkvist O, Johansson SH. Perichondrial arthroplasty. A clinical study in twenty-six patients. Scand J Plast Reconstr Surg 1980;14 (1):71-87

12 Czitrom AA, Keating S, Gross AE. The viability of articular cartilage in fresh osteochondral allografts after clinical transplantation. J Bone Joint Surg Am 1990;72(4):574-581

13 Gomoll AH, Farr J, Gillogly SD, Kercher J, Minas T. Surgical management of articular cartilage defects of the knee. J Bone Joint Surg Am 2010;92(14):2470-2490

14 Slutsky DJ, Nagle DJ. Wrist arthroscopy: current concepts. J Hand Surg Am 2008;33(7):1228-1244

15 Culp R, Osterman AL, Kaufmann RA. Wrist arthroscopy: operative procedures. In: Green DP, Hotchkiss RN, Pederson WC, Wolfe SW, et al. Green's Operative Hand Surgery. 5th ed. Philadelphia: Elsevier; 2005:781-803
16 Boulas HJ, Herren A, Büchler U. Osteochondral metatarsophalangeal autografts for traumatic articular metacarpophalangeal defects: a preliminary report. J Hand Surg Am 1993;18(6): 1086-1092

17 Gaul JS Jr. Articular fractures of the proximal interphalangeal joint with missing elements: repair with partial toe joint osteochondral autografts. J Hand Surg Am 1999;24(1):78-85

18 Lo CY, Chang YP. Osteochondral grafting of the metacarpophalangeal joint in rheumatoid arthritis. J Hand Surg [Br] 2003;28(1):94-97

19 Ozyurekoglu T. Multiple osteochondral autograft transfer to the proximal interphalangeal joint: case report. J Hand Surg Am 2010;35(6):931-935

20 Mehin R, Giachino AA, Backman D, Grabowski J, Fazekas A. Autologous osteoarticular transfer from the proximal tibiofibular joint to the scaphoid and lunate facets in the treatment of severe distal radial fractures: a report of two cases. J Hand Surg Am 2003;28(2):332-341

21 Merrell GA, Barrie KA, Wolfe SW. Sigmoid notch reconstruction using osteoarticular graft in a severely comminuted distal radius fracture: a case report. J Hand Surg Am 2002;27(4):729-734

22 Del Piñal F, García-Bernal FJ, Delgado J, Sanmartín M, Regalado J. Reconstruction of the distal radius facet by a free vascularized osteochondral autograft: anatomic study and report of a patient. J Hand Surg Am 2005;30(6):1200-1210

23 Lee YK, Lee M, Lee JM. Osteochondral autograft transplantation for osteochondritis dissecans of the scaphoid: case report. J Hand Surg Am 2011;36(5):820-823 